\title{
ADOLESCÊNCIA, ÁLCOOL E DROGAS: UMA REVISÃO NA PERSPECTIVA DA PROMOÇÃO DA SAÚDE.
}

\author{
Adolescence, alcohol and drugs: A reflection in the Health Promotion \\ perspective. \\ Adolescencia, alcohol y drogas: Una reflexión en la perspectiva \\ de la promoción de la salud.
}

Maria Beatriz de Paula Tavares Cavalcante ${ }^{1}$

Maria Dalva Santos Alves 2

Maria Grasiela Teixeira Barroso ${ }^{3}$

\section{RESUMO}

É durante a adolescência que o jovem forma sua personalidade e individualidade, e é também o período em que as drogas se fazem mais presentes. Com este estudo, objetivou-se desenvolver uma análise crítica sobre a necessidade de ações educativas na prevenção do uso de drogas entre adolescentes, verificando os fatores de risco a ele relacionados. Estudo de caráter reflexivo, no qual constatamos que a Segunda Conferência Internacional para a Promoção da Saúde foi pioneira ao introduzir o tema como prioritário na elaboração de políticas públicas. Estudos associam fatores socioeconômicos e envolvimento familiar como fatores de risco para o consumo de álcool e outras drogas. Entende-se que seja fundamental ajudá-los na vivência dessa fase de transição, destacando a família e a educação como primordiais em suas formações como sujeitos rumo à promoção da saúde.

Palavras-chave: Adolescência. Promoção da Saúde. Enfermagem.

\begin{abstract}
During the adolescence the young form their own personality and individuality, and this is the period that the drugs make it selves more present. This study objective to develop a critical analysis on the necessity of educative actions in the prevention of the drug's consumption between adolescents and being verified the risk factors related to them. This is a reflective article in which we evidenced that the Second International Conference for the Health Promotion was pioneering when introducing the subject as priority in the elaboration of public politics. Studies associate social and economics factors, and practical familiar as risk factors for the alcohol consumption and other drugs. It was understood that is basic to help them in the experience of this transition's phase, detaching family and education as primordial in their formations as citizens, in the route to the health promotion.
\end{abstract}

Keywords: Adolescence. Health Promotion. Nursing.

\section{Resumen}

En el período de adolescencia el joven forma su personalidad e individualidad y es una época en que las drogas están mas presentes. Con los objetivos de desarrollar un análisis crítico sobre la necesidad de acciones educativas en la prevención del uso de drogas entre ellos y verificar los factores de riesgo relacionados. El estudio reflexivo tuvo apoyo en la Segunda Conferencia Internacional para la Promoción de la Salud que indica la introducción del tema como prioridad en la planificación de las políticas públicas. Estudios asocian los factores sociales y económicos así como las relaciones familiares como factores de riesgo para el consumo de alcohol y otras drogas. Es fundamental ayudarlos, destacando la importancia de la familia y educación como de grande relevancia en sus vidas así como formar sujetos dirigidos a la promoción de la salud.

Palabras clave: Adolescencia. Promoción de la Salud Enfermería.

1 Estudante de graduação em Enfermagem na Universidade Federal do Ceará, bolsista PIBIC- CNPq, ${ }^{2}$ Professora Doutora Associada I do Departamento de Enfermagem a Universidade Federal do Ceará, ${ }^{3}$ Professora Doutora Emérita da Universidade Federal do Ceará, pesquisadora de produtividade do CNPq - Orientadora. 


\section{INTRODUÇÃO}

A adolescência é um período crítico na vida de cada indivíduo, pois nessa fase o jovem vivencia descobertas significativas e afirma a personalidade e a individualidade. Caracterizar a adolescência somente como faixa etária seria uma maneira muito simplista de observá-la, uma vez que ela compreende a transformação do jovem até a idade adulta, não apenas sob o ponto de vista biológico, mas também social e, principalmente, psicológico.

Nessa fase, o conceito de interação grupal é perceptível, e o adolescente busca pertencer a um grupo com o qual se identifica. Este terá a capacidade de influenciar suas ações e fará com que adote atitudes as quais serão a prova de sua aceitação na "tribo".

Justamente nesse período, em que o grupo de amigos atinge importância social principal, os conflitos familiares atingem 0 pico, fazendo com que os pais percam um pouco do seu poder de controle sobre os filhos, que buscam a imagem de adulto independente no grupo de amigos no qual estão inseridos, o que é uma tendência natural dos adolescentes. É principalmente nesse período de crise que as drogas entram em suas vidas.

Nosso papel, como profissionais da saúde, é alertar aos pais para que se aproximem de seus filhos nessa fase tão conturbada de suas vidas, destacando sempre a importância da família e da manutenção de uma convivência familiar saudável, em suas formações. Cabe aos pais ensiná-los a distinguir entre o certo e o errado, fazendo-se presentes em qualquer que seja o caminho tomado pelo filho.

Para a Enfermagem, o estudo sobre o comportamento dos adolescentes perante as drogas é de fundamental importância, uma vez que é de nosso conhecimento o fato de que tanto as medidas preventivas como as estatísticas disponíveis em nosso País são insuficientes para tratar e dimensionar a problemática. Como enfermeiros, cuidadores e promotores da saúde, devemos nos aproximar da realidade dos nossos jovens a fim de conhecer o problema e elaborar políticas públicas e programas de prevenção e tratamento para o uso/abuso de álcool e drogas, visando sempre à manutenção de uma boa qualidade de vida desses adolescentes longe das drogas.

Com este artigo, objetivamos desenvolver uma análise crítica sobre a necessidade de ações educativas na prevenção do uso de drogas entre adolescentes, verificando os fatores de risco a eles relacionados.

\section{ABORDAGEM METODOLÓGICA}

Trata-se de um estudo de caráter reflexivo que, segundo a Escola Anna Nery Revista de Enfermagem, caracteriza-se por analisar aspectos teóricos e/ou construções teóricas da Enfermagem ou áreas afins, oriundas de processo reflexivo, discernimento e consideração atenta dos autores, que poderão contribuir para o aprofundamento de temas profissionais.

A pesquisa foi realizada entre os dias 13 e 26 de agosto de 2007, sendo utilizados como referenciais artigos, manuais e livros produzidos sobre a temática em estudo, bem como a
Internet, onde foram utilizados os descritores: adolescentes, álcool, drogas, enfermagem e riscos.

Do total de referenciais teóricos utilizados para a feitura do artigo, 40\% foram textos extraídos de revistas como Revista de Saúde Pública (13,3\%), Escola Anna Nery Revista de Enfermagem (20\%) e Cadernos de Saúde Pública (6,7\%); $40 \%$, de livros e $20 \%$, de manuais produzidos pelo Ministério da Saúde e por este em parceria com entidades como a UNESCO.

\section{REVISÃO DA LITERATURA}

0 estudo sobre a adolescência tem se aprofundado cada vez mais em virtude da preocupação que estes suscitam às autoridades governamentais, principalmente nas áreas da saúde e da educação. Este termo, que significa o período de vida iniciado na puberdade, e que acaba quando o jovem entra no que, culturalmente, se considera a idade adulta, é caracterizado por intensas transformações. É durante essa fase que o indivíduo se desenvolve física e emocionalmente e adota comportamentos influenciados pelo meio sócioambiental.

A geração atual é considerada a mais urbana da história; entretanto, à medida que a urbanização possibilita cada vez mais o acesso à educação e aos serviços de saúde, os adolescentes são mais expostos aos riscos de uso de drogas lícitas e ilícitas. Vários fatores se associam ao abuso de álcool na adolescência, a começar pelos aspectos sócio-históricos, como a industrialização e a urbanização de décadas recentes e a crise econômica dos anos 1980, responsável pela dificuldade de inserção do jovem no mercado de trabalho e a conseqüente insatisfação de suas necessidades. Não se pode subestimar, também, a crescente produção industrial de bebidas alcoólicas e o forte apelo dos meio de comunicação em favor do consumo por indivíduos de todas as classes sociais ${ }^{1}$.

Fatores de risco para uso de drogas entre adolescentes no Brasil são pouco estudados, sendo a maior parte das informações disponíveis a esse respeito provenientes de estudos realizados em outros países. Além de fatores sociodemográficos (sexo, idade, classe social), os estudos indicam associação do uso de drogas com envolvimento parental ou familiar no consumo de álcool ou drogas, não criação por ambos os pais, baixa percepção de apoio paterno e materno, amigos que usam drogas, ausência de prática religiosa, bem como menor freqüência à prática de esportes².

0 apelo dos meios de comunicação, que estimula o consumo de drogas lícitas, como álcool e tabaco, assim como a aceitação social e condescendência familiar para o consumo destas drogas, parecem creditar em sua utilização a idéia de rito de passagem para a vida adulta 3 .

Além do estímulo constante dos meios de comunicação e da condescendência dos pais, podemos mencionar outros fatores de risco que viabilizam o acesso dos adolescentes a essas substâncias, como sua grande disponibilidade, principalmente de drogas lícitas, em estabelecimentos comerciais e a falta de fiscalização adequada para sua venda, sendo comum a compra por menores de 18 anos; as normas 
sociais, que estimulam o hábito de "beber socialmente" ou fumar por "ser elegante"; o baixo preço de algumas dessas drogas, o que torna sua aquisição possível à maioria da população; e, por fim, em conflitos familiares graves, quando o adolescente se utiliza desse artifício como fuga à situação $0^{3,4}$. 0 risco generalizado parece, assim, definir e circunscrever negativamente esse período da vida, dando ensejo a expressões, ações e atitudes absurdas em relação aos adolescentes ${ }^{5}$.

À nossa sociedade pertencem idéias sobre a adolescência que se associam à noção de crise, desordem, irresponsabilidade; um problema social a ser resolvido, que merece atenção pública 5 .

0 consumo de álcool em excesso pelo adolescente traz várias conseqüências graves para sua saúde, evidenciando-se que esta droga socialmente aceita é a porta de entrada para o consumo e o vício em outras drogas, ditas ilícitas. Estudos apontam que o consumo de álcool entre adolescentes acontece, em média, aos 11 anos de idade, e que é comum o primeiro contato acontecer na presença da família, o que traz responsabilidades para este, como a orientação e educação para a saúde de seus filhos, enfatizando os riscos e perdas decorrentes do consumo de bebidas espirituosas. A família, assim como o relacionamento familiar, é um elemento essencial que deve ser mais bem observado e abordado pela Enfermagem. Esta deve, por sua vez, trabalhar na orientação para que este relacionamento se desenvolva de forma saudável, orientando os pais no sentido de sua participação efetiva na educação dos filhos, estabelecendo uma relação baseada na confiança e o exemplo, com uma conduta, em relação ao álcool, de serenidade e equilíbrio. Tudo isso contribui para a proteção de seus filhos no que se refere ao uso de álcool e outras drogas, prevenindo conseqüências deletérias para suas saúdes e colaborando para uma qualidade de vida cada vez melhor ${ }^{3}$.

De acordo com Gonçalves ${ }^{6}$, droga é toda substância que, ao ser introduzida, inalada, ingerida ou injetada, provoca alterações no funcionamento do organismo, modificando suas funções. Há um grupo de drogas que possui a capacidade de atuar no psiquismo, as denominadas psicotrópicas, que provocam alteraç̃̃es do humor, percepção, sensações de prazer e euforia, alívio, medo, dor etc. É particularmente a esse grupo que se refere ao utilizar o termo droga.

Sobre o estudo da ação das drogas no organismo humano, Patrício ${ }^{7}$, já em 1999, dizia que todas as substâncias chamadas de drogas psicotrópicas têm efeitos no sistema nervoso, produzindo no homem resultados psicoativos. Estas podem ser classificadas em: psicolépticos (sedativos), psicoanalépticos (estimulantes), psicodislépticos (perturbadores), ou, ainda, efeitos combinados ou potenciados. Podem ser divididas em naturais, semi-sintéticas ou sintéticas; lícitas ou ilícitas. Do ponto de vista sociocultural, podem ser socialmente integradas ou rejeitadas; de finalidade terapêutica ou não. Por último, essas substâncias podem provocar ou não dependência física. Em sua maioria, porém, produzem dependência psicológica ${ }^{7}$.
Essa dependência psicológica e a limitação que o uso contínuo traz aos seus usuários preocupam autoridades e pais, que buscam afastar seus filhos desse vício. Na tentativa de mostrar à sociedade que é preciso combater esse problema com maior afinco e elaborar estratégias para ajudar esses dependentes químicos a se restabelecerem e reintegrarem à comunidade, as autoridades em saúde incluíram nas cartas para a promoção da saúde este item.

A Segunda Conferência Internacional sobre Promoção da Saúde, em Adelaide, Austrália, identificou quatro áreas prioritárias para promover ações imediatas em políticas públicas saudáveis. Entre elas, configurou o uso de tabaco e álcool, descritos como dois grandes riscos à saúde, que merecem imediata atenção dentro da perspectiva das políticas públicas voltadas à saúde. As cartas para a Promoção da Saúde não só têm o interesse em elaborar políticas públicas saudáveis como também em promover a responsabilidade social para com a saúde ${ }^{8}$.

A Declaração de Adelaide é precisa em asseverar que o tabaco não só traz malefícios para quem fuma, mas também para quem convive com ele, os chamados fumantes passivos. Em relação ao produto álcool, a Declaração de Adelaide 0 conceitua como contribuinte para distúrbios sociais e traumas físicos e mentais ${ }^{8}$. Sem desconsiderar os dados estatísticos que apontam o etilismo como responsável por um conjunto de danos que se manifestam em todas as dimensões do viver humano, seja na forma de baixa produtividade seja na de acidentes de trânsito ou delinqüências, é na família que suas conseqüências mais danosas se manifestam. Em Adelaide, os governos foram alertados para o elevado e crescente potencial humano perdido por doenças, incapacidades e mortes causadas pelo consumo de drogas, como o uso de álcool e tabaco ${ }^{8}$.

Algumas questões se mostram relevantes, quando nos referimos à vulnerabilidade dos adolescentes no plano individual, social ou pragmático. 0 uso e o abuso de álcool e outras drogas constituem as principais causas desencadeadoras de situações de vulnerabilidade na adolescência, a exemplo dos acidentes, suicídios, violência, gravidez não planejada e a transmissão de doenças por via sexual e endovenosa, nos casos das drogas injetáveis 5 .

Recente pesquisa mostrou ser no início da adolescência que os jovens brasileiros usam drogas pela primeira vez. Embora igualmente precoce, o consumo de drogas ilícitas só costuma ocorrer em média um ano e meio depois da primeira tragada ou do primeiro copo, aos 14,9 anos .

0 uso de álcool (bebida espirituosa) entre adolescentes é, naturalmente, um tema controverso no meio social e acadêmico brasileiro. Ao mesmo tempo em que a lei brasileira define como proibida a venda de bebidas alcoólicas para menores de 18 anos, é prática muito comum o consumo de álcool pelos jovens - seja no domićlio, em festividades, ou mesmo em ambientes públicos. A sociedade como um todo adota atitudes paradoxais ante o tema: por um lado, condena o abuso de álcool pelos jovens, mas é tipicamente permissiva ao estímulo do consumo por meio da propaganda. 
A promoção da saúde do adolescente é objeto de debates, tanto na área acadêmica como nas instituições de saúde e educação. A principal preocupação é no sentido de estimular nos adolescentes comportamentos e estilos de vida saudáveis que insiram no eixo de motivação para o autocuidado.

Em um estudo realizado com escolares e educadores em uma escola pública, em São Paulo, o uso de drogas foi freqüentemente problematizado pelos coordenadores, associando-o à falta de lazer e às condições de vida dos jovens, enquanto o etilismo foi considerado de maneira específica, relacionado mais freqüentemente aos pais dos alunos. Portanto, o grupo social em que os jovens estão inseridos parece contribuir para a adoção desse hábito ${ }^{10}$. Entendemos que a família é 0 locus onde 0 adolescente vê seus principais exemplos de vida, motivo pelo qual consideramos necessário o acompanhamento e a atuação da Enfermagem nessa instituição.

É fundamental ajudar os adolescentes na compreensão e vivência dessa fase de transição para a vida adulta, valorizandoos como sujeitos da sua história, destacando a família e a escola como espaços primordiais para formar a opinião desses sujeitos no sentido de promoção da saúde. Ressaltamos aqui a importância dos enfermeiros como agentes-chave no processo de transformação social, participando no desenho e na implantação de programas e projetos de promoção de saúde, prevenção do uso e abuso de álcool e outras drogas e interação social ${ }^{11}$.

Moraes, Leitão e Braga ${ }^{12: 93}$ referem que:

As políticas de saúde não estão atuando eficazmente em situações de reabilitação de jovens; sem falar que os poucos resultados positivos alcançados pelos serviços especializados em reabilitação esbarram na complexidade que envolve a realidade socioeconômica, a exclusão social, 0 desemprego, a falta de perspectiva e a violência, condições identificáveis na vida dos adolescentes.

Essa problemática tem amplo alcance, envolvendo não só o adolescente, como também sua família e seu contexto socioeconômico e cultural. Por essa complexidade, pode-se dizer que nem toda ação de intervenção vá ter o efeito final almejado. A prevenção mostra-se como uma das formas mais eficazes de lidar com o uso e 0 abuso de drogas, principalmente entre os adolescentes. A precaução não deve se limitar a ações isoladas, mas desenvolver-se em todas as frentes, enfatizandose a orientação e mobilização desses adolescentes, enfocando ações de redução de danos, reabilitação e socialização desses jovens.

Desenvolver ações de atenção à saúde dos adolescentes difere da assistência clínica individual e da simples informação ou repressão. 0 Ministério da Saúde ${ }^{5: 10}$ preconiza que:

0 modelo a ser desenvolvido deve permitir uma discussão sobre as razões da adoção de um comportamento preventivo e o desenvolvimento de habilidades que permitam a resistência às pressões externas, a expressão de sentimentos, opiniões, dúvidas, inseguranças, medos e preconceitos, de forma a dar condições para o enfrentamento e a resolução de problemas e dificuldades do dia-adia.

A Educação em Saúde apresenta-se aqui como ferramenta de ação do enfermeiro para fazer com que a informação chegue até seu destino final, o adolescente, conscientizando-o acerca da manutenção de um estilo de vida saudável. Varela, Silva e Barroso ${ }^{13: 16}$ configuram a educação para a saúde como sendo:

(...) o processo de qualificação do indivíduo (inserido em grupo historicamente situado), para a convivência social harmoniosa e o exercício da cidadania em todos os niveis e contextos em que interage norteando-se pelos valores humanos e utilizando princípios e instrumentos democráticos visando à promoção da qualidade de vida no planeta.

Analisando a situação, como fizeram Barroso, Vieira e Varela ${ }^{14: 34}$ em seu estudo, precisamos utilizar estratégias didáticas que modifiquem a maneira de interação dos adolescentes inseridos na sociedade, ampliando a capacidade de compreensão da complexidade dos determinantes de ser saudável, a fim de que possam atingir a promoção da saúde.

Os autores ${ }^{3,4,11}$ são claros ao acentuar a necessidade de elaboração e, principalmente, implementação de programas que visem à promoção da saúde e à prevenção de danos aos adolescentes, que enfoquem a detecção precoce de fatores de risco para o uso/abuso de drogas lícitas e ilícitas, e viabilizem o acesso dos adolescentes aos serviços de saúde e à informação. Para tanto, a capacitação do enfermeiro é imprescindível, permitindo que a categoria amplie seu conhecimento científico sobre a temática e que adquira novas ferramentas para trabalhar em prol de uma abordagem mais dinâmica e interdisciplinar dos problemas que vivenciará, procurando sempre envolver não só o adolescente, como também a sociedade de sua pertença, como família, escola, os parceiros, pais e amigos.

Nesse sentido, políticas públicas são desenvolvidas para o adolescente, garantindo seu acesso às ações de promoção da saúde e prevenção de doenças, atenções a agravos e doenças, e reabilitação, respeitando os princípios do Sistema Único de Saúde (SUS) ${ }^{15}$.

0 principal desafio na implementação das políticas elaboradas é a captação do público-alvo, trazer o adolescente para as unidades de saúde, a fim de otimizar o contato deste com a equipe de saúde e assim disponibilizar os serviços de apoio, bem como facilitar o acesso à informação. Quando a captação desses jovens não ocorre de maneira satisfatória no interior das unidades, cabe à equipe desenvolver atividades estratégicas extramuros na comunidade. 0 enfermeiro apresenta-se como membro atuante na equipe, uma vez que sua formação acadêmica lhe proporciona subsídios para tanto. Ações como a divulgação interna na unidade, visitas domiciliares, divulgaç̃̃es na comunidade e estabelecimento 
de parcerias institucionais com famílias, associações juvenis, grupos sociais e religiosos, clubes e escolas, são fundamentais para que um maior número de adolescentes seja envolvido e informado sobre as perdas e ganhos, quando se escolhe ou se abdica das drogas ${ }^{4,15}$.

\section{CONSIDERAÇÕES FINAIS}

0 estudo deixa claras a necessidade da participação do enfermeiro e da sua intervenção em favor dos adolescentes, dos familiares e das instituiç̃oes educativas de que fazem parte, no controle do uso de drogas. A Enfermagem precisa intervir junto a essas instituições para promover a saúde desses adolescentes e prevenir quanto ao uso de álcool e drogas. Para tanto, o enfermeiro deve atuar, de maneira interdisciplinar, com outras profissões da saúde e instâncias sociais, praticando a transdisciplinaridade e a intersetorialidade, sensibilizando os adolescentes para as causas e conseqüências do problema, num entendimento biopsicossocial, oferecendo informações sobre substâncias psicoativas e os problemas relacionados ao uso, almejando sempre obter os melhores resultados para 0 seu cliente.

Todo o nosso desafio reside em desenvolver atividades educativas e de conscientização, que recobrem, principalmente, a valorização do sentido da vida por esses adolescentes.

\section{Referências}

1.Muza GM, Bettiol H, Muccillo G, Barbieri MA. Consumo de substâncias psicoativas por adolescentes escolares de Ribeirão Preto, SP (Brasil). I-Prevalência do consumo por sexo, idade e tipo de substância. Rev Saude Publica $1997 \mathrm{fev}$; 31 (1): 21-29

2.Tavares BF, Béria JU, Lima MS. Fatores associados ao uso de drogas entre adolescentes escolares. Rev Saúde Pública 2004 dez; 38(6): 787-96.

3.Alavarse GMA, Carvalho MDB. Álcool e adolescência: o perfil de consumidores de um município do norte do Paraná. Esc Anna Nery Rev Enferm 2006 dez; 10(3): 408-16.

4.Almeida Filho AJ, Ferreira MA, Gomes MLB, Silva RC, Santos TCF. 0 adolescente e as drogas: conseqüências para a saúde. Esc Anna Nery Rev Enferm 2007 dez; 11(4): 605-10.

5.Ministério da Saúde (BR) Secretaria de Atenção à Saúde. Área de Saúde do Adolescente e do Jovem. Marco legal: saúde, um direito de

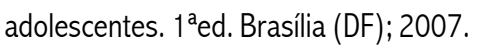

6.Gonçalves EC. Alguns conceitos referentes à toxicomania. In: Bucher R. As drogas e a vida: uma abordagem psicossocial. São Paulo (SP): EPU, 1998.

7.Patrício LDB. Abuso de drogas na Europa: reflexão rumo ao ano 2000. Mundo da Saúde 1999 jan/fev 23(1): 56-63

8.Ministério da Saúde (BR). Projeto Promoção da Saúde: Declaração de Alma-Ata. Carta de Ottawa. Declaração de Adelaide. Declaração de Sundsvall. Declaração de Santafé de Bogotá. Declaração de Jacarta. Rede de Megapaíses e Declaração do México. Brasilia (DF; 2001.

9.Castro MG, Abramovay M. Drogas nas escolas. São Paulo (SP): UNESCO-DST/AIDS-MS-CNPQ; 2002.
10.Soares CB, Salvetti MG, Ávila LK. Opinião de escolares e educadores sobre saúde: o ponto de vista da escola pública de uma região periférica do Município de São Paulo. Cad Saude Publica. 2003 jul/ ago; 19(4): 1153-61.

11. Gonçalves SSPM, Tavares CMM. Atuação do enfermeiro na atenção ao usuário de álcool e outras drogas nos serviços extra-hospitalares. Esc Anna Nery Rev Enferm 2007 dez; 11(4): 586-92.

12. Moraes LMP, Leitão GCM, Braga VAB. Uso de drogas por adolescentes: construção de conceitos. In: Souza AMA, Braga VAB, Fraga MNO. Saúde, saúde mental e suas interfaces. Fortaleza (CE): Pós-Graduação DENF/UFC/FFOE, FCPC; 2002. p. 93-100.

13.Varela ZMV, Silva RM, Barroso, MGT. Dimensões do cotidiano: violência doméstica, saúde da mulher e desempenho no trabalho. Fortaleza (CE): Ed UFC; 1998.

14.Barroso MGT, Vieira NFC, Varela ZMV. Educação em saúde no contexto da promoção humana. Fortaleza (CE): Ed Demócrito Rocha; 2003.

15.Ministério da Saúde(BR). Secretaria da Atenção à Saúde. Saúde integral de adolescentes e jovens: orientações para a organização de serviços de saúde. Brasilia (DF); 2005. 